\title{
Fiber-chip edge coupler with large mode size for silicon photonic wire waveguides
}

\author{
Martin Papes, ${ }^{1, *}$ Pavel Cheben, ${ }^{2,8}$ Daniel Benedikovic, ${ }^{3}$ Jens H. Schmid, ${ }^{2}$ James Pond, ${ }^{4}$ \\ Robert Halir, ${ }^{5}$ Alejandro Ortega-Moñux, ${ }^{5}$ Gonzalo Wangüemert-Pérez, ${ }^{5}$ Winnie N. Ye, ${ }^{6}$ \\ Dan-Xia Xu, ${ }^{2}$ Siegfried Janz, ${ }^{2}$ Milan Dado, ${ }^{7}$ and Vladimír Vašinek ${ }^{1}$ \\ ${ }^{1}$ Technical University of Ostrava, Faculty of Electrical Engineering and Computer Science, 17. listopadu 15/2172, \\ 70833 Ostrava-Poruba, Czech Republic \\ ${ }^{2}$ National Research Council Canada, 1200 Montreal Road, Ottawa, Ontario, K1A 0R6, Canada \\ ${ }^{3}$ Institut d'Electronique Fondamentale, Université Paris Sud, CNRS, UMR 8622, Université Paris-Saclay, Bat. 220, \\ Orsay Cedex, France \\ ${ }^{4}$ Lumerical Solutions, Inc., 300 - 535 Thurlow St., Vancouver, V6E 3L2, Canada \\ ${ }^{5}$ ETSI Telecomunicación, Universidad de Málaga, 29010 Málaga, Spain \\ ${ }^{6}$ Carleton University, Department of Electronics, 1125 Colonel By Dr., Ottawa, ON, K1S 5B6, Canada \\ ${ }^{7}$ University of Zilina, Dept. of Telecommunication and Multimedia, Univerzitna 8215/1, 01026 Zilina, Slovakia \\ 8pavel.cheben@nrc.ca \\ "martin.papes@vsb.cz
}

\begin{abstract}
Fiber-chip edge couplers are extensively used in integrated optics for coupling of light between planar waveguide circuits and optical fibers. In this work, we report on a new fiber-chip edge coupler concept with large mode size for silicon photonic wire waveguides. The coupler allows direct coupling with conventional cleaved optical fibers with large mode size while circumventing the need for lensed fibers. The coupler is designed for $220 \mathrm{~nm}$ silicon-on-insulator (SOI) platform. It exhibits an overall coupling efficiency exceeding $90 \%$, as independently confirmed by 3D FiniteDifference Time-Domain (FDTD) and fully vectorial 3D Eigenmode Expansion (EME) calculations. We present two specific coupler designs, namely for a high numerical aperture single mode optical fiber with $6 \mu \mathrm{m}$ mode field diameter (MFD) and a standard SMF-28 fiber with $10.4 \mu \mathrm{m}$ MFD. An important advantage of our coupler concept is the ability to expand the mode at the chip edge without leading to high substrate leakage losses through buried oxide (BOX), which in our design is set to $3 \mu \mathrm{m}$. This remarkable feature is achieved by implementing in the $\mathrm{SiO}_{2}$ upper cladding thin high-index $\mathrm{Si}_{3} \mathrm{~N}_{4}$ layers. The $\mathrm{Si}_{3} \mathrm{~N}_{4}$ layers increase the effective refractive index of the upper cladding near the facet. The index is controlled along the taper by subwavelength refractive index engineering to facilitate adiabatic mode transformation to the silicon wire waveguide while the Siwire waveguide is inversely tapered along the coupler. The mode overlap optimization at the chip facet is carried out with a full vectorial mode solver. The mode transformation along the coupler is studied using 3DFDTD simulations and with fully-vectorial 3D-EME calculations. The couplers are optimized for operating with transverse electric (TE) polarization and the operating wavelength is centered at $1.55 \mu \mathrm{m}$.
\end{abstract}

(C)2016 Optical Society of America

OCIS codes: (130.0130) Integrated optics; (050.6624) Subwavelength structures; (130.3120) Integrated optics devices; (230.7370) Waveguides.

\section{References and links}

1. A. Barkai, A. Liu, D. Kim, R. Cohen, N. Elek, H.-H. Chang, B. H. Malik, R. Gabay, R. Jones, M. Paniccia, and N. Izhaky, "Efficient mode converter for coupling between fiber and micrometer size silicon waveguides," in Proceedings of 4th IEEE International Conference on Group IV Photonics (IEEE, 2007), pp. 1-3. 
2. R. Soref, "The past, present, and future of silicon photonics," IEEE J. Sel. Top. Quantum Electron. 12(6), 16781687 (2006).

3. D. Taillert, W. Bogaerts, P. Bienstman, T. Krauss, P. Van Daele, I. Moerman, S. Verstuyft, K. De Mesel, and R. Baets, "An out-of-plane grating coupler for efficient butt-coupling between compact planar waveguides and single-mode fibers," IEEE J. Quantum Electron. 38(7), 949-955 (2002).

4. A. Davidson, I. Glesk, and A. Buis, "Has silicon reached it's limit?" Adv. Electr. Electron. Eng. 12(6), 590-598 (2014).

5. D. Benedikovic, P. Cheben, J. H. Schmid, D.-X. Xu, J. Lapointe, S. Wang, R. Halir, A. Ortega-Monux, S. Janz, and M. Dado, "High-efficiency single etch step apodized surface grating coupler with subwavelength structure," Laser Photonics Rev. 8(6), 93-97 (2014).

6. R. Halir, P. Cheben, S. Janz, D.-X. Xu, I. Molina-Fernández, and J. G. Wangüemert-Pérez, "Waveguide grating coupler with subwavelength microstructures," Opt. Lett. 34(9), 1408-1410 (2009).

7. C. Alonso-Ramos, P. Cheben, A. Ortega-Moñux, J. H. Schmid, D.-X. Xu, and I. Molina-Fernández, "Fiber-chip grating coupler based on interleaved trenches with directionality exceeding 95\%," Opt. Lett. 39(18), 5351-5354 (2014).

8. P. Markov, J. G. Valentine, S. M. Weiss, F. X. Karter, C. Porzier, A. Roman, J.-M. Fedeli, R. Gabay, R. Jones, M. Paniccia, and N. Izhaky, "Fiber-to-chip coupler designed using an optical transformation," Opt. Express 20(13), 14705-14713 (2012).

9. V. R. Almeida, R. R. Panepucci, and M. Lipson, "Nanotaper for compact mode conversion," Opt. Lett. 28(15), 1302-1304 (2003).

10. P. Cheben, D.-X. Xu, S. Janz, and A. Densmore, "Subwavelength waveguide grating for mode conversion and light coupling in integrated optics," Opt. Express 14(11), 4695-4702 (2006).

11. S. Romero-Garcia, B. Marzban, F. Merget, R. B. Shen, N. Elek, H.-H. Chang, B. H. Malik, R. Gabay, R. Jones, M. Paniccia, and N. Izhaky, "Edge couplers with relaxed alignment tolerance for pick-and-place hybrid integration of III-V lasers with SOI waveguides," IEEE J. Sel. Top. Quantum Electron. 20(4), 369-379 (2014).

12. T. Barwicz, A. Janta-Polczynski, M. Khater, Y. Thibodeau, R. Leidy, J. Maling, S. Martel, S. Engelmann, J. S. Orcutt, P. Fortier, and W. M. J. Green, "An O-band metamaterial converter interfacing standard optical fibers to silicon nanophotonic waveguides," in Optical Fiber Communication Conference, OSA technical Digest (online) (Optical Society of America, 2015), paper Th3F.3.

13. P. Cheben, P. J. Bock, J. H. Schmid, J. Lapointe, S. Janz, D. X. Xu, A. Densmore, A. Delâge, B. Lamontagne, T. J. Hall, and T. J. Hall, "Refractive index engineering with subwavelength gratings for efficient microphotonic couplers and planar waveguide multiplexers," Opt. Lett. 35(15), 2526-2528 (2010).

14. B. Bakir, A. G. De Vazquez, R. Orobtchouk, P. Lyan, C. Porzier, A. Roman, J.-M. Fedeli, R. Gabay, R. Jones, M. Paniccia, and N. Izhaky, "Low-loss and polarization-insensitive edge fiber couplers fabricated on 200-mm silicon-on-insulator wafers," IEEE Photonics Technol. Lett. 22(11), 739-741 (2010).

15. A. Khilo, M. A. Popović, M. Araghchini, F. X. Kärtner, C. Porzier, A. Roman, J.-M. Fedeli, R. Gabay, R. Jones, M. Paniccia, and N. Izhaky, "Efficient planar fiber-to-chip coupler based on two-stage adiabatic evolution," Opt. Express 18(15), 15790-15806 (2010).

16. R. Takei, E. Omoda, M. Suzuki, S. Manako, T. Kamei, M. Mori, and Y. Sakakibara, "Ultra narrow silicon inverse taper waveguide fabricated with double-patterning photolithography for low-loss spot-size converter," Appl. Phys. Express 5(5), 052202 (2012).

17. Q. Fang, T.-Y. Liow, J. F. Song, C. W. Tan, M. B. Yu, G. Q. Lo, and D.-L. Kwong, "Suspended optical fiber-towaveguide mode size converter for silicon photonics," Opt. Express 18(8), 7763-7769 (2010).

18. M. Tokushima, A. Kamei, and T. Horikawa, "Dual-tapered 10- $\mu \mathrm{m}$-spot-size converter with double core for coupling polarization-independent silicon rib waveguides to single-mode optical fibers," Appl. Phys. Express 5, $22202(2012)$

19. T. Tsuchizawa, K. Yamada, H. Fukuda, T. Watanabe, J.-I. Takahashi, M. Takahashi, T. Shoji, E. Tamechika, S.I. Itabashi, and H. Morita, "Microphotonic devices based on silicon microphotonic technology, IEEE J. Selected Topics in Quant," Electron. 11(1), 232-240 (2005).

20. A. W. Snyder and J. Love, Optical Waveguide Theory (Chapman and Hall, 1983).

21. Photon Design [online]. Available: www.photond.com.

22. Lumerical [online]. Available: www.lumerical.com.

23. R. Halir, P. Bock, P. Cheben, A. Ortega-Monux, C. Alonso-Ramos, J. H. Schmid, J. Lapointe, D.-X. Xu, J. G. Wanguemert-Perez, I. Molina-Fernandez, and S. Janz, "Waveguide sub-wavelength structures: a review of principles and applications," Laser Photonics Rev. 9(1), 25-49 (2015).

24. S. M. Rytov, "Electromagnetic properties of a finely stratified medium," Sov. Phys. 2(3), 466-475 (1956).

25. L. Zavargo-Peche, A. Ortega-Monux, J. G. Wanguemert-Perez, and I. Molina-Fernandez, "Fourier based combined techniques to design novel sub-wavelength optical integrated devices," Prog. Electromagnetics Res. 123, 447-465 (2012).

26. P. Cheben, J. H. Schmid, S. Wang, D.-X. Xu, M. Vachon, S. Janz, J. Lapointe, Y. Painchaud, and M.-J. Picard, "Broadband polarization independent nanophotonic coupler for silicon waveguides with ultra-high efficiency," Opt. Express 23(17), 22553-22563 (2015).

27. M.-J. Picard, Y. Painchaud, C. Latrasse, C. Larouche, F. Pelletier, and M. Poulin, "Novel spot-size converter for optical fiber to sub- $\mu \mathrm{m}$ silicon waveguide coupling with low loss, low wavelength dependence and high tolerance to alignment," in 41st European Conference on Optical Communications, (IEEE, 2015), pp. 1-3. 


\section{Introduction}

Silicon-on-insulator (SOI) is a prominent platform for monolithic integration of optical and electrical devices, largely benefiting from the high-volume manufacturing technologies available in the microelectronics industry. The high refractive index contrast between the silicon and silicon dioxide $(\Delta \mathrm{n} \sim 2)$ in SOI allows the realization of highly compact integrated optical devices with sub-micrometer waveguide dimensions [1-3]. However, coupling of light between the SOI waveguides and optical fibers poses a challenge due to the mode size mismatch, given the mode size for a standard optical fiber (SMF-28) is about two orders of magnitude larger compared to a typical silicon wire waveguides.

Two types of approaches have been extensively investigated to alleviate this problem, specifically edge couplers and surface grating couplers. Surface grating couplers diffract the light to or from the planar waveguide facing the optical fiber positioned at near-vertical incidence above the chip [4-7]. An important advantage of surface grating couplers is that the light can be coupled to and from the chip at any location on the chip, facilitating wafer-scale testing. A notable drawback is the dispersive operating principle of grating couplers that limits their operating bandwidth and also typically makes them polarization sensitive.

Fiber-chip edge couplers can provide a substantially increased operating bandwidth. Inverse taper geometries are often used, where the light is coupled between the optical fiber and a narrow waveguide tip which expands the mode. This delocalized mode near the chip facet is then adiabatically transformed to the highly confined waveguide mode by gradually increasing the waveguide width [8-10]. However, achieving a mode size similar to that of a standard optical fiber is challenging in SOI waveguides, since the mode expands symmetrically around the narrow waveguide core, but yet has to remain confined within the buried oxide (BOX) layer in order to prevent mode leakage into the silicon substrate. In fact, the mode leakage to the substrate through the BOX layer is the main obstacle to achieving large mode sizes. A limited mode size is a disadvantage since lensed fibers are required for high coupling efficiencies and precise (sub-micron) fiber-chip alignment is demanded [11,12]. An inverse taper structure with a large mode size has been reported [12] based on the subwavelength grating (metamaterial) concept [13], but it requires local etching of the silicon substrate under the coupler. In order to increase the mode size without increasing the BOX thickness or locally etching the substrate, the waveguide mode can be allowed to expand preferentially into the upper cladding. This can be achieved by increasing the upper cladding refractive index. An interesting approach was demonstrated by Ben Bakir et al. [14] using an injector waveguide with a fundamental mode matching the optical fiber mode. The injector waveguide comprises a $3.5-\mu \mathrm{m}$-thick layer of silicon-rich oxide with a specific refractive index that is controlled by the amount of silicon nanocrystals. Khilo et al. [15] reported a coupler based on combination of rib and inverse tapers. First, the light is coupled into a lowindex rib waveguide and the rib is then gradually tapered down and a high-index inverse taper is introduced. Alternatively, the polyimide secondary waveguide in combination with an ultranarrow tip [16] can be used, however, at the expense of increased fabrication complexity. While mode coupling and the phase-matching condition between the injector waveguides may limit the operating wavelength range in some devices [14], this does not constitute a general limitation and several wavevelength-insensitive inverse taper edge couplers have been reported [17-19].

In this work, we present a new type of fiber-chip edge coupler with a large mode size. Specifically, we show that by engineering the effective refractive index of the upper cladding using thin high-index layers, a mode overlap exceeding $94 \%$ can be achieved at the chip facet, circumventing the need for an increased bottom oxide thickness or local substrate etching. Furthermore, since the edge coupler concept utilizes the mode evolution principle, a broadband operation is achieved. 


\section{Coupling efficiency}

The coupling efficiency of an edge coupler can be expressed as the product of two main factors: the overlap between the optical fiber mode and the mode at the coupler tip near the chip facet $\left(\eta_{1}\right)$, and the efficiency of transformation of the delocalized mode at the facet to the mode of the nominal interconnecting waveguide $\left(\eta_{2}\right)$. For quasi-TE and quasi-TM polarizations, where one of the components of the electrical field is dominant, the modal overlap can be calculated as [20]:

$$
\eta_{1}=\frac{\left|\int E_{1} E_{2} d A\right|^{2}}{\int\left|E_{1}\right|^{2} d A \int\left|E_{2}\right|^{2} d A},
$$

where $\mathrm{E}_{1}$ and $\mathrm{E}_{2}$ are the complex electric field amplitudes of the waveguide mode (at the chip facet) and the optical fiber mode, respectively. The mode transformation efficiency $\eta_{2}$ depends on the specifics of the taper structure which is used to transform the mode at the chip facet to the mode of the interconnecting waveguide.

The design procedure is performed is two steps. First, we optimize the transverse field overlap between the optical fiber mode and the waveguide mode at the chip facet. In the seond step, we design the longitudinal taper structure which transforms the delocalized mode near the facet to the mode of the silicon wire interconnecting waveguide.

\section{Mode field overlap optimization}

The cross-section schematics of the two considered coupler structures near the chip facet are shown in Figs. 1 and 2. The couplers are designed for an SOI substrate with a 220-nm-thick silicon waveguide layer and a $3-\mu \mathrm{m}$-thick BOX layer. The upper cladding comprises a stack of $\mathrm{Si}_{3} \mathrm{~N}_{4}$ and $\mathrm{SiO}_{2}$ layers. The corresponding material refractive indexes are: $\mathrm{n}_{\mathrm{Si}}=3.476, \mathrm{n}_{\mathrm{SiO} 2}$ $=1.444$, and $\mathrm{n}_{\mathrm{Si} 3 \mathrm{~N} 4}=2.016$ near $1550 \mathrm{~nm}$ wavelength.

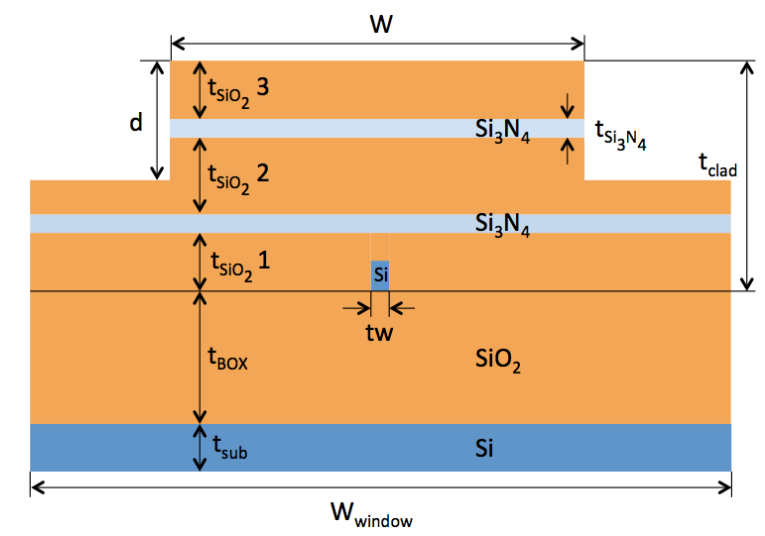

Fig. 1. Cross-section schematics of the first coupler design near the chip facet. 


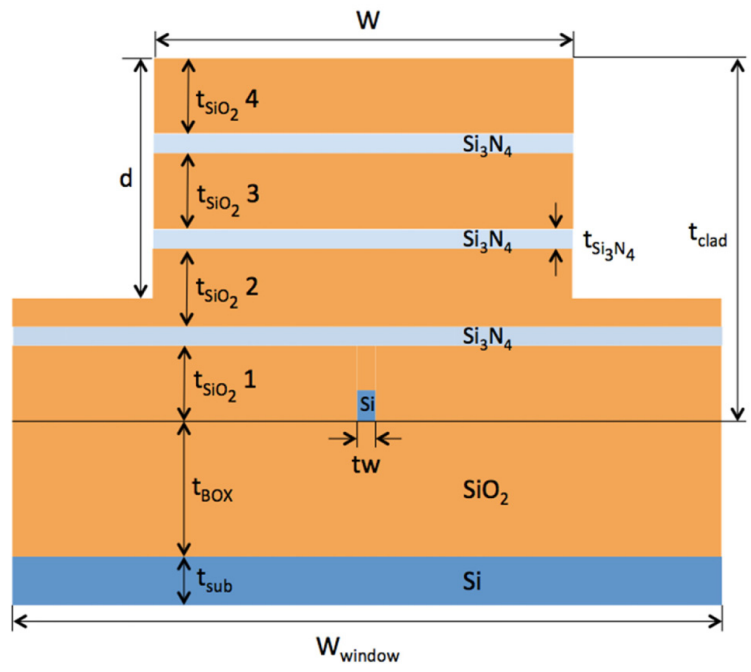

Fig. 2. Cross-section schematics of the second coupler design near the chip facet.

Our coupler concept is based on mode delocalization effect by reducing the width of the silicon waveguide core, while judiciously positioning the $\mathrm{Si}_{3} \mathrm{~N}_{4}$ layers and controlling their optical properties to maximize the field overlap with the optical fiber mode and minimize the mode coupling to the silicon substrate. The $\mathrm{Si}_{3} \mathrm{~N}_{4}$ layers increase the effective refractive index of the upper cladding, therefore modifying the mode spatial distribution by effectively pulling it towards the upper cladding. The mode size at the chip facet is directly controlled by the cladding thickness $\left(\mathrm{t}_{\mathrm{clad}}\right)$, the etch depth (d), and the rib width (w) (see Figs. 1 and 2). The mode field overlap calculations are carried out by using a full-vectorial mode solver, FIMMWAVE from Photon Design [21].

Our first coupler design (Fig. 1) incorporates a 5- $\mu \mathrm{m}$-thick upper cladding. The structure is designed for coupling with a high numerical aperture (HNA) optical fiber with a $6-\mu \mathrm{m}$ mode field diameter (MFD). We study first the HNA coupler design as it gives an advantage in terms of alignment tolerances compared to tapered/lensed fibers with smaller MFDs, while potentially yielding high coupling efficiency. Indeed, coupling with standard SMF-28 optical fiber (10.4 $\mu \mathrm{m}$ MFD; Design 2) would be an ideal solution. However, using HNA fiber can be a good compromise between tapered/lensed fibers and SMF-28 fibers. The HNA fibers are readily available from vendors and can easily be spliced with SMF-28 fiber with negligible loss. The reference structure comprises a 3- $\mu \mathrm{m}$-thick BOX layer, a silicon wire waveguide with a dimension of $150 \mathrm{~nm} \times 220 \mathrm{~nm}$, and a 5- $\mu \mathrm{m}$-thick upper $\mathrm{SiO}_{2}$ cladding layer. A rib waveguide geometry with an etch depth $d=3 \mu \mathrm{m}$ is used to laterally confine the mode, as it yields a maximum mode overlap with the optical fiber mode in the horizontal direction. According to our mode overlap calculations, the optimal rib width is $8.75 \mu \mathrm{m}$. The thickness of the $\mathrm{SiO}_{2}$ layer between the two $\mathrm{Si}_{3} \mathrm{~N}_{4}$ layers is $2.23 \mu \mathrm{m}$. The $1.63-\mu \mathrm{m}$-thick oxide layer is used to separate the bottom $\mathrm{Si}_{3} \mathrm{~N}_{4}$ layer from the BOX layer, and the 1.03- $\mu$ m-thick $\mathrm{SiO}_{2}$ layer is used on top of the the upper $\mathrm{Si}_{3} \mathrm{~N}_{4}$ layer. Figure 3(a) shows the fundamental quasi-TE polarized mode supported by this optimized waveguide structure. It can be observed that the mode field is noticeably pulled into the upper cladding. It has to be highlighted that due to the integral nature of the mode overlap (Eq. (1), the "spikes" in the waveguide mode have little impact on the overall coupling efficiency. The calculated MFDs of the rib waveguide are 4.77 $\mu \mathrm{m}$ and $6.6 \mu \mathrm{m}$ in the vertical (y) and horizontal (x) directions, respectively. The overlap with the Gaussian fiber mode ( $6 \mu \mathrm{m}$ MFD) is calculated as a 2D integral according to Eq. (1). The dominant E-field component $\left(\mathrm{E}_{\mathrm{x}}\right)$ of the waveguide mode is extracted directly from a mode solver, whereas the fiber mode is expressed as a Gaussian, with offsets $\left(\mathrm{x}_{0}\right.$ and $\left.\mathrm{y}_{0}\right)$ in the 
horizontal and vertical directions. These offsets are swept to determine the optimum position of the fiber, resulting in $\mathrm{x}_{0}=17.5 \mu \mathrm{m}, \mathrm{y}_{0}=6.1 \mu \mathrm{m}$, and a field overlap of $94.5 \%$. The alignment tolerance for a $1 \mathrm{~dB}$ coupling efficiency penalty was found to be $1.3 \mu \mathrm{m}$ for this design.

Our second design is optimized for coupling to a standard optical fiber (SMF-28), with a MFD of $10.4 \mu \mathrm{m}$. In this design, we use an 11- $\mu \mathrm{m}$-thick upper cladding and the rib height (d) is $6.5 \mu \mathrm{m}$. The optimal rib width yelding a maximum mode overlap with the SMF-28 mode is $14.15 \mu \mathrm{m}$. The $\mathrm{SiO}_{2}$ thickness between the three $\mathrm{Si}_{3} \mathrm{~N}_{4}$ layers is $2.72 \mu \mathrm{m}$, with the same $\mathrm{SiO}_{2}$ thickness used between the bottom $\mathrm{Si}_{3} \mathrm{~N}_{4}$ layer and the BOX, and above the upper $\mathrm{Si}_{3} \mathrm{~N}_{4}$ layer. The calculated profiles of the fundamental quasi-TE polarized mode of this waveguide structure are shown in Fig. 3(b). Similarly as for the HNA structure (Design 1), it is observed that the mode field distribution is shifted towards the upper cladding. The calculated MFDs of the waveguide mode are $10.8 \mu \mathrm{m}$ for the horizontal and $8.4 \mu \mathrm{m}$ for the vertical directions. The optimum offset for the fiber mode is found to be $\mathrm{x}_{0}=17.5 \mu \mathrm{m}, \mathrm{y}_{0}=9.3 \mu \mathrm{m}$, resulting in a field overlap of $92.6 \%$. For a $1 \mathrm{~dB}$ coupling efficiency penalty the large mode field affords a relaxed alignment tolerance of $2.2 \mu \mathrm{m}$.

The optimized waveguide transverse cross-section parameters for the two considered coupler designs are summarized in Table 1.

Table 1. Optimized waveguide parameters for the two considered coupler designs.

\begin{tabular}{|c|c|c|}
\hline Dimension & Design 1 & Design 2 \\
\hline $\mathbf{t}_{\mathrm{BOX}}[\boldsymbol{\mu m}]$ & 3 & 3 \\
\hline $\mathbf{t}_{\mathrm{SiO} 2} 1[\mu \mathrm{m}]$ & 1.63 & 2.72 \\
\hline $\mathbf{t}_{\mathrm{SiO} 2} 2[\mu \mathrm{m}]$ & 2.23 & 2.72 \\
\hline $\mathrm{t}_{\mathrm{SiO} 2} 3[\mu \mathrm{m}]$ & 1.03 & 2.72 \\
\hline$t_{\mathrm{SiO} 2} 4[\mu \mathrm{m}]$ & - & 2.72 \\
\hline $\mathbf{t}_{\text {clad }}[\boldsymbol{\mu m}]$ & 5 & 11 \\
\hline $\mathbf{t}_{\mathrm{Si3N} \mathbf{N}}[\mathrm{nm}]$ & 20 & 30 \\
\hline $\mathbf{d}[\mu \mathrm{m}]$ & 3 & 6.5 \\
\hline $\mathbf{w}[\mu \mathrm{m}]$ & 8.75 & 14.15 \\
\hline tw $[\mathrm{nm}]$ & 150 & 150 \\
\hline
\end{tabular}

The use of a multilayer patterned overlay structure indeed carries the price of increased fabrication complexity; however, the layer materials and patterning processes are in principle straightforward and CMOS compatible. Furthermore, the overlay structure is required only locally at the chip edge, where the couplers are situated and could be removed elsewhere on the chip. Therefore it does not limit the potential for further optical-electrical device integration which may require metal layers on the upper cladding.
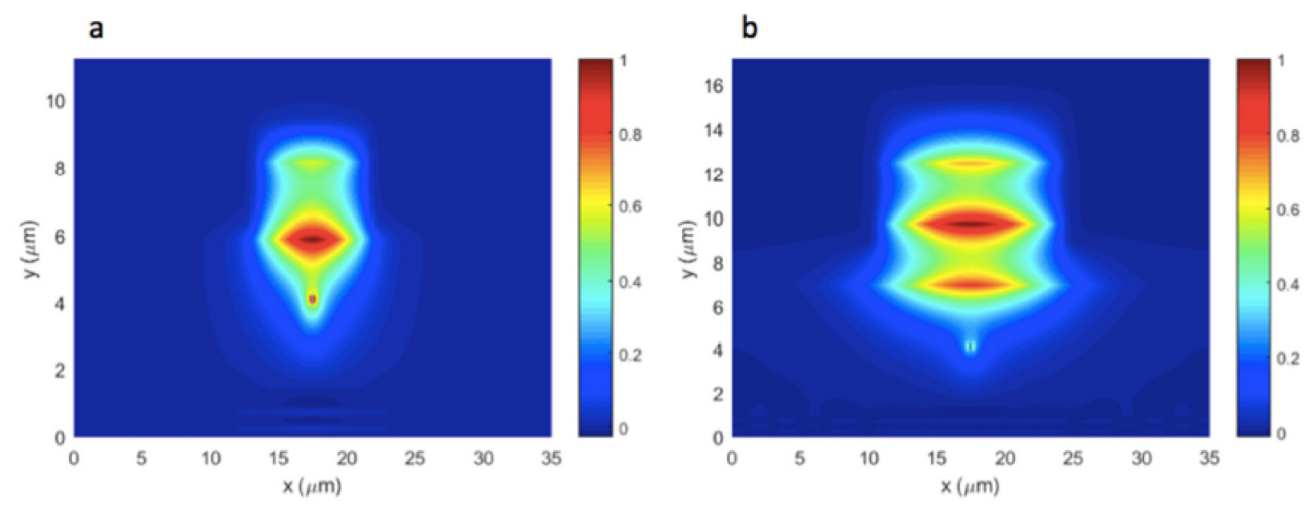

Fig. 3. Fundamental TE mode at the coupler tip for the two considered coupler designs, namely for a) a high numerical aperture fiber with $6 \mu$ m mode size, and b) a standard SMF-28 fiber design with $10.4 \mu \mathrm{m}$ mode size. 


\section{Mode transformer design}

\subsection{Finite-Difference Time-Domain (3D-FDTD) simulation}

The tapered structure, schematically shown in Fig. 4, transforms the delocalized mode supported by the rib waveguide geometry at the chip facet to the highly confined mode of the silicon wire waveguide. The rib waveguide geometry is used in combination with an inverse taper and thin $\mathrm{Si}_{3} \mathrm{~N}_{4}$ layers with engineered refractive index along the mode propagation direction. The mode transforming structure was designed using three-dimensional FiniteDifference Time-Domain (3D-FDTD) and Eigenmode Expansion (3D-EME) simulators, from Lumerical [22]. Because of the high computational demands for the coupling structure for SMF-28 fiber (Fig. 2), the 3D-FDTD simulations were carried out only for the edge coupler design for HNA fiber (Fig. 1). The 3D-EME simulations were carried out for both designs.

The optimized structure for coupling with HNA fiber comprises two 20-nm-thick $\mathrm{Si}_{3} \mathrm{~N}_{4}$ layers embedded in silica cladding, formed over an inversely tapered silicon waveguide. According to our 3D-FDTD calculations, the optimal coupling is achieved for the silicon waveguide core tapered from $150 \mathrm{~nm}$ at the chip facet to the waveguide width of $270 \mathrm{~nm}$ along a coupling length of $260 \mu \mathrm{m}$, as it is schematically shown on Fig. 4.

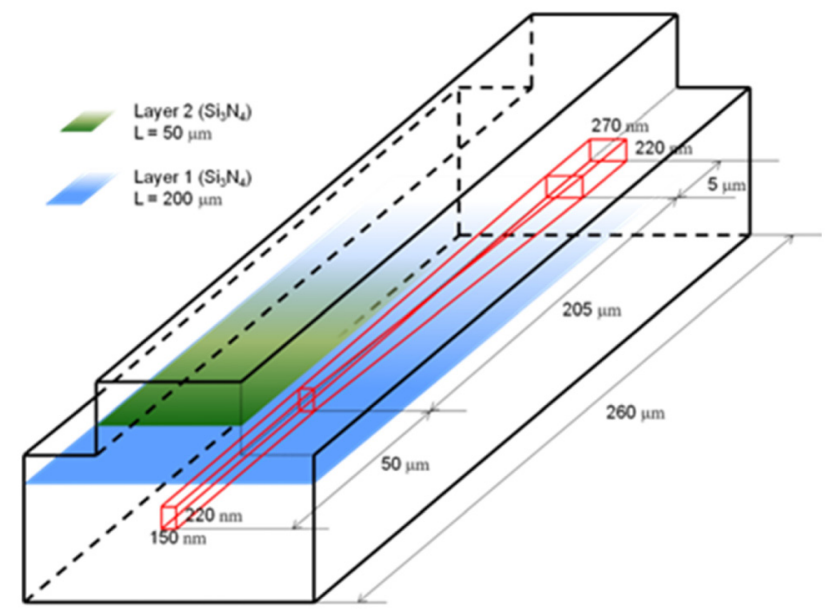

Fig. 4. Schematic of the fiber-chip edge coupler. Design 1, for HNA fiber.

It should be noticed that $270 \mathrm{~nm}$ is the width of the inversely tapered waveguide at the coupler termination point, at which the light is fully coupled from the over-layer structure to the silicon waveguide underneath. In practical implementations of the coupler, this $270 \mathrm{~nm}$ wide $\mathrm{Si}$ waveguide is to be connected to the nominal Si waveguide width (typically $450 \mathrm{~nm}$ for 220-nm-thick SOI waveguides). For this purpose, we assume that the Si wire width at the coupler termination point is tapered to the nominal width of the nominal waveguide. Since such tapered waveguides are commonly used in integrated optics and their performance is well understood with efficiencies approaching $100 \%$, the effect on the coupler performance was assumed to be practically negligible and we did not specifically include it in our simulations.

The effective refractive index of $\mathrm{Si}_{3} \mathrm{~N}_{4}$ layers is controlled by sub-wavelength grating engineering. The sub-wavelength grating (SWG) is implemented as a periodic array of highindex segments with varying duty ratio along the light propagation direction. Specifically, the subwavelength grating comprises alternated strips of the highe index $\left(\mathrm{Si}_{3} \mathrm{~N}_{4}\right)$ and the low index $\left(\mathrm{SiO}_{2}\right)$ materials, as schematically shown in Fig. 5. 


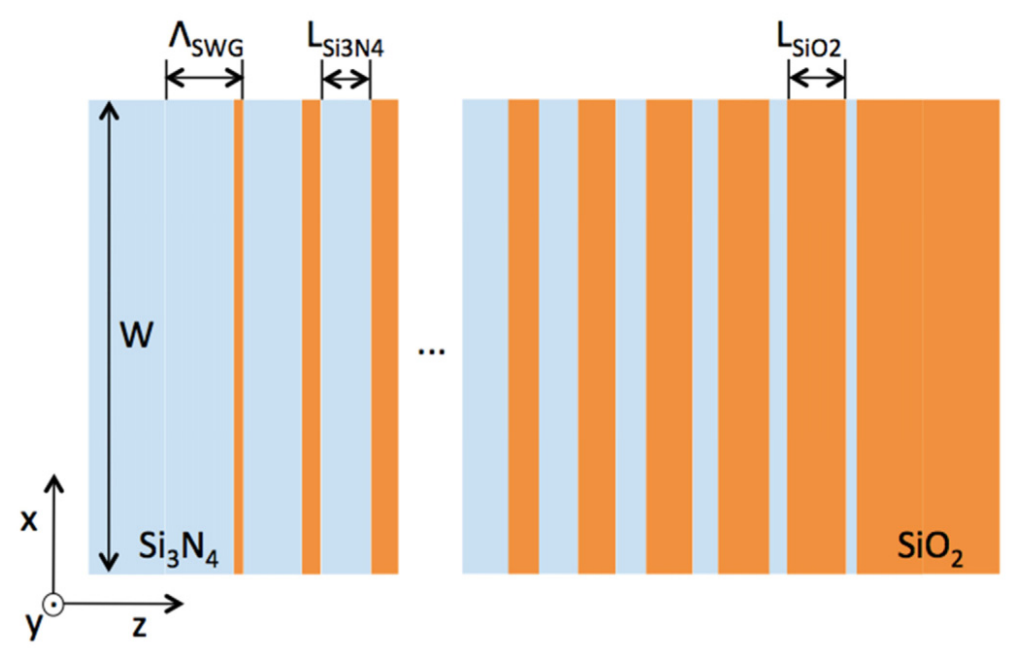

Fig. 5. In-plane schematic (top view) of the sub-wavelength engineered $\mathrm{Si}_{3} \mathrm{~N}_{4} / \mathrm{SiO}_{2}$ layer.

According to the effective medium theory (EMT) [24], by controlling the sub-wavelength grating duty cycle $\mathrm{DC}$, an equivalent homogeneous medium can be synthesized with an intermediate refractive index $n_{\mathrm{SWG}}\left(\mathrm{n}_{\mathrm{Si} 3 \mathrm{~N} 4}>\mathrm{n}_{\mathrm{SWG}}>\mathrm{n}_{\mathrm{SiO} 2}\right)$. Here, the DC is defined as the ratio between the high-index $\mathrm{Si}_{3} \mathrm{~N}_{4}$ strip of length $\mathrm{L}_{\mathrm{Si} 3 \mathrm{~N} 4}$ and the grating period $\Lambda_{\mathrm{SWG}}$. Provided that the grating period is less than the first-order Bragg period $\left(\Lambda_{\mathrm{SWG}}<\Lambda_{\mathrm{Bragg}}=\lambda_{0} / 2 \mathrm{n}_{\mathrm{eff}}\right)$, the grating operates in the sub-wavelength regime and the diffraction effects are suppressed [2326]. In our coupler, the effective medium refractive index gradually decreases from the value of $2.016\left(\mathrm{Si}_{3} \mathrm{~N}_{4}\right)$ near the chip facet to $1.444\left(\mathrm{SiO}_{2}\right)$ at the points, where the $\mathrm{Si}_{3} \mathrm{~N}_{4}$ layers effectively terminate. The SWG engineering allows an additional design degree of freedom and improved performance compared to previously reported designs with high-index overlays [27]. According to our 3D-FDTD calculations, the optimal lengths of the two $\mathrm{Si}_{3} \mathrm{~N}_{4} / \mathrm{SiO}_{2}$ strips are found to be $50 \mu \mathrm{m}$ and $200 \mu \mathrm{m}$, respectively. In both the 3D-FDTD and the 3DEME simulations (see section 4.2) we represent the SWG layers as homogenous layers with varying refractive index, to alleviate the computational load. The sub-wavelength grating pitch of $500 \mathrm{~nm}$ and $20-\mathrm{nm}$-thick $\mathrm{Si}_{3} \mathrm{~N}_{4}$ layers are assumed. The geometry of the subwavelength grating that implements the desired gradual decrease in the refractive index is found using the following procedure: First, we simulate the fundamental Bloch mode propagating in the structure shown in the inset of Fig. 6(a), and obtain its effective index as a function of the SWG duty-cycle. This calculation is virtually exact, because the only approximation is to assume that the width of the SWG layer $(\mathrm{w}=8.75 \mu \mathrm{m})$ is much larger than its thickness $(20 \mathrm{~nm})$, which indeed is the case. In a second step, we obtain the equivalent refractive index of the core of a homogeneous slab waveguide (see inset in Fig. 6(b)) that yields a fundamental slab mode with the same effective index as the Bloch mode in Fig. 6(a). The approximations inherent to this second step are: i) we consider only moderate wavelength variations and ii) the field profile propagating through the SWG in the 3D case locally resembles the fundamental Bloch mode. The latter indeed is the case as the other guiding structures are spaced a few micrometers apart. The data in Fig. 6(a) can thus be used as a look-up table to find the SWG geometry that implements a certain equivalent refractive index. For Design 2, the procedure is identical except for the corresponding change in the high-index layers thickness of $30 \mathrm{~nm}$. 
(a)

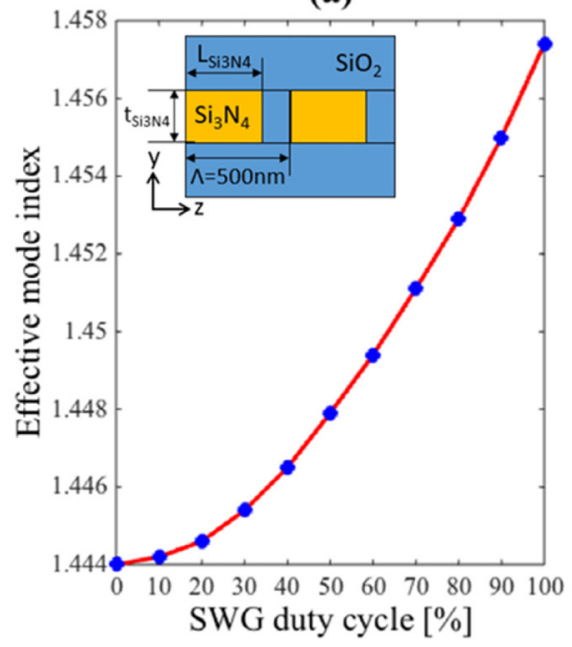

(b)

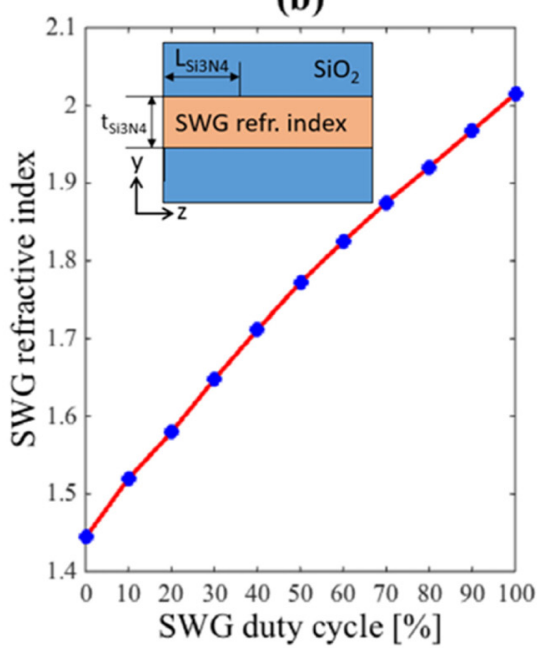

Fig. 6. a) Calculation of the equivalent refractive index of the SWG structure as a function of grating duty-cycle $\left(\mathrm{L}_{\mathrm{Si} 3 \mathrm{~N} 4} / \Lambda\right)$. a) Effective index of the fundamental Bloch mode propagating through the $\mathrm{SWG}$ engineered $\mathrm{Si}_{3} \mathrm{~N}_{4} / \mathrm{SiO}_{2}$ layer as a function of its duty-cycle (see inset). b) Equivalent refractive index in the core of homoegenous slab waveguide (see inset) that yields a fundamental slab mode with the same effective index as the Bloch mode in Fig. 6(a).

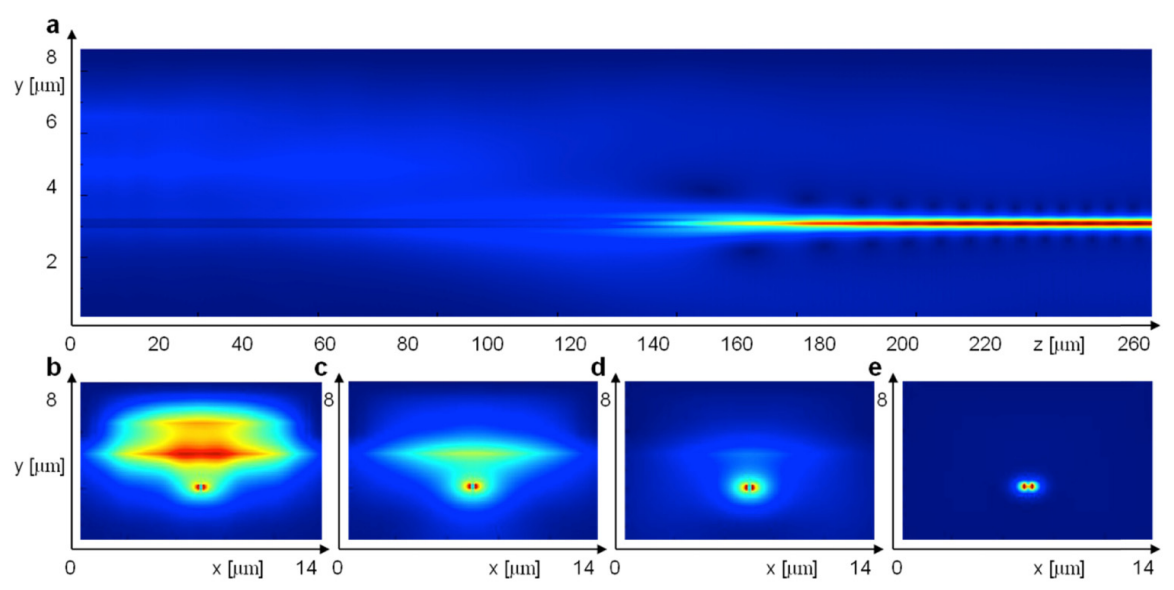

Fig. 7. Calculated modal field evolution along the coupler. a) Electric field evolution along the coupler (side view, field component $\mathrm{E}_{\mathrm{x}}$ ). Transverse mode distributions $\mathrm{b}$ ) near the chip facet, at c) $40 \mu \mathrm{m}$ and d) $80 \mu \mathrm{m}$ from the facet, and e) highly-confined mode in the Si-wire waveguide positioned $260 \mu \mathrm{m}$ from the facet. Quasi-TE polarization, wavelength $\lambda=1550 \mathrm{~nm}$.

The 3D-FDTD simulation results are presented in Fig. 7. The evolution of the electric field along the coupler is shown in Fig. 7(a). It is observed a smooth transition between the delocalized mode near the facet (left side) and the highly confined silicon wire mode (right side). Panels (b-e) in Fig. 7 show the transverse mode distributions at different positions along the coupler, namely at the chip facet $(\mathrm{z}=0)$, at two intermediate positions $(\mathrm{z}=40 \mu \mathrm{m}$ and 80 $\mu \mathrm{m})$, and at the junction with the interconnecting waveguide $(\mathrm{z}=260 \mu \mathrm{m})$. The calculated mode conversion efficiency is $96 \%$. The overall coupling efficiency including the mode overlap at the facet is $90.7 \%$. This corresponds to an overall coupling loss of $0.42 \mathrm{~dB}$. 


\subsection{EME simulations}

While 3D-FDTD simulations are highly accurate they are computationally intensive for larger structures. For edge coupler design and optimization, the 3D-EME method is appealing because the simulations are relatively fast (typically a few minutes or less on a single workstation) and, once the modes of the structure and relevant overlap integrals are calculated, it is possible to recalculate results for any length of taper with a negligible computational cost. In addition, the EME method can immediately provide the coefficients of all forward and backward propagating modes in each section of the taper, making it easy to determine where the rate of tapering should be adjusted to preserve the adiatatic limit of mode conversion. In FDTD, the same information requires additional post-simulation analysis, the computational cost of which is comparable to the entire EME simulation.

The 3D-EME results are compared with the 3D-FDTD results for the the HNA fiber design (Design 1) in Fig. 8. We compare the transmission to the fundamental mode of the output waveguide in both cases as a function of the total taper length, where the total tapered structure is scaled linearly from the nominal length of $255 \mu \mathrm{m}(50 \mu \mathrm{m}$ and $205 \mu \mathrm{m}$, respectively, for the first and second tapered sections, as shown in Fig. 4) to the length shown in the Fig. 8(c). Convergence testing was performed for both simulation methods. We can see that the field profiles and transmission results are in excellent agreement. It is observed that the nominal taper length of $255 \mu \mathrm{m}$ can achieve better than $95 \%$ efficiency for $\eta_{2}$. This efficiency is even higher than was found in the initial design simulations which were run on a more coarse mesh and a smaller transverse simulation size. It is worth noting that beyond approximately $300 \mu \mathrm{m}$, the total transmission actually starts to decrease. Both simulation results (FDTD and EME) shown in Fig. 8 include the Si substrate and therefore account for the leakage losses due to the finite BOX thickness, thus confirming one of the key features of this design which is the ability to expand the mode at the chip edge without leading to high substrate leakage losses. The transverse simulation dimensions are $20 \mu \mathrm{m} \times 9.2 \mu \mathrm{m}$, and the smallest spatial mesh size used in the transverse direction is $20 \mathrm{~nm}$. The $\mathrm{Si}_{3} \mathrm{~N}_{4} / \mathrm{SiO}_{2} \mathrm{SWG}$ is represented as a $30 \mathrm{~nm}$ thick layer of a single material with a refractive index that varies continuously from that of $\mathrm{Si}_{3} \mathrm{~N}_{4}$ to that of $\mathrm{SiO}_{2}$. For EME, the mesh size can be easily reduced to $5 \mathrm{~nm}$ or less in certain sensitive regions, for example around the tapered silicon material, but in FDTD there is a significant computational penalty for reducing the mesh size in any region due to the Courant-Friedrichs-Lewy (CFL) condition which requires a smaller time step when smaller spatial grids are used to preserve numerical stability. In both simulation methods a conformal mesh is used to improve convergence at larger mesh sizes. Furthermore, in the EME method, a specific treatment of the tapered sections is required to avoid nonphysical reflections that can occur from staircasing effects. This treatment, called the continuously varying cross-sectional (CVCS) method [22] makes it possible to calculate the modes at a relatively small number of cross sections in the tapered regions (typically 10 to 20). 

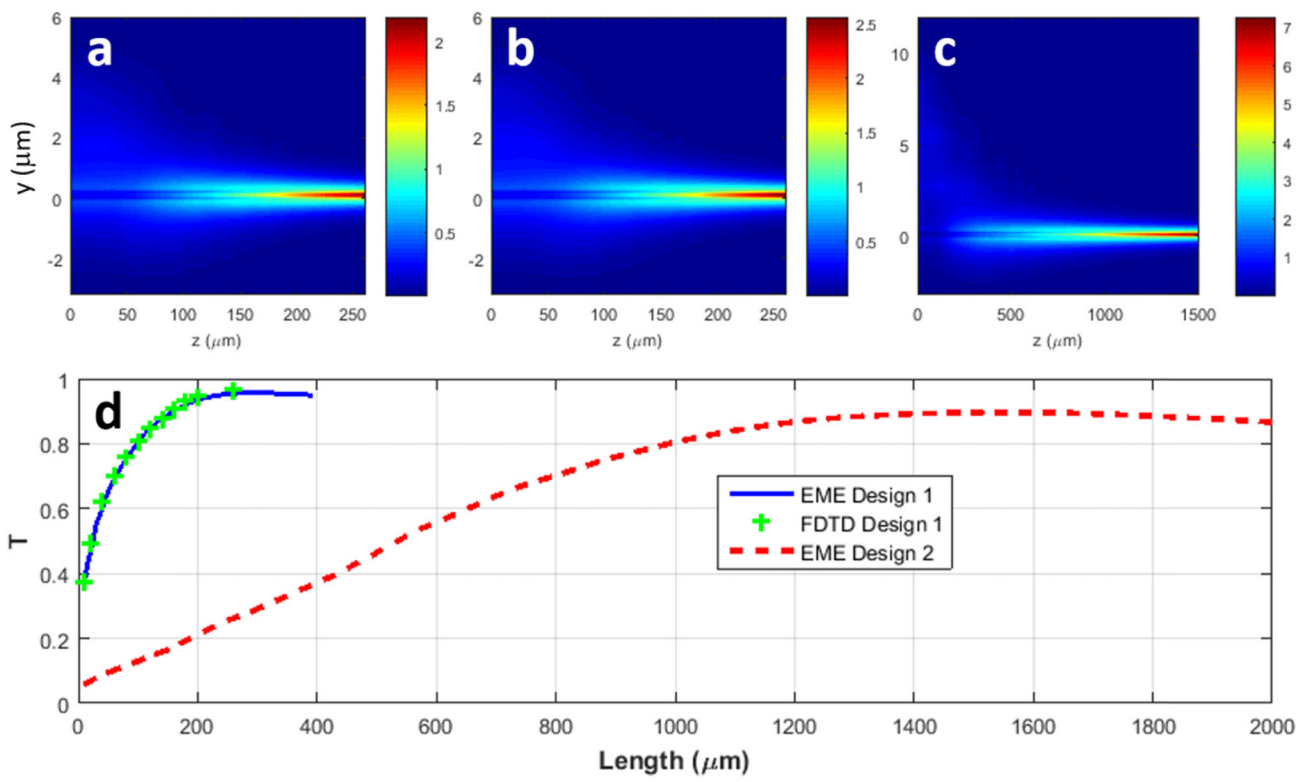

Fig. 8. The electric field evolution along the coupler as calculated by a) FDTD for Design 1 (HNA), b) EME for Design 1 (HNA), and c) EME for Design 2 (SMF-28). d) The comparison of transmission to the fundamental mode as a function of length for Design 1 (EME and FDTD) and Design 2 (EME). For Design 1, the entire curve from $10 \mu \mathrm{m}$ to $400 \mu \mathrm{m}$ with 100 different lengths can be calculated in a few minutes on a single workstation with EME, while the FDTD simulations range from a few minutes (at the shortest lengths) to many hours for 260 $\mu \mathrm{m}$ length on a single workstation. For Design 2, the structure is too large to be simulated by 3D-FDTD on typical workstations. All simulations include the Si substrate.

We also calculated the mode transformation efficiency for the coupler without the SWG pattering, that is for homogeneous (continuous) $\mathrm{Si}_{3} \mathrm{~N}_{4}$ layers. For this case, the calculated efficiency is reduced to $91 \%$, resulting in a $0.23 \mathrm{~dB}$ penalty on the coupler overall efficiency. For applications when a simple fabrication process is preferred and this moderate penalty in the efficiency can be tolerated, homogeneous $\mathrm{Si}_{3} \mathrm{~N}_{4}$ layers can be used, omitting the SWG patterning step. This comparatively small difference in coupling efficiencies for the coupler with and without the SWG structure also means that the coupler performance is substantially robust to fabrication imperfections in the SWG patterning step and that the requirements on the minimum SWG feature size are relaxed.

Figure 9 shows the magnitude of the forward propagating mode coefficients as a function of position for the HNA fiber design (Design 1) for the nominal structure shown in Fig. 4. The design is clearly close to the adiabatic limit where light propagates only in the fundamental mode (mode 1) which has a coefficient magnitude that is close to 1 at all positions. The color bar is intentionally oversaturated to emphasize where the light couples into higher order modes so that we can identify the regions where this coupling occurs. Further design improvements could be achieved by adjusting the rate of tapering in these regions to further reduce coupling to higher order modes. 


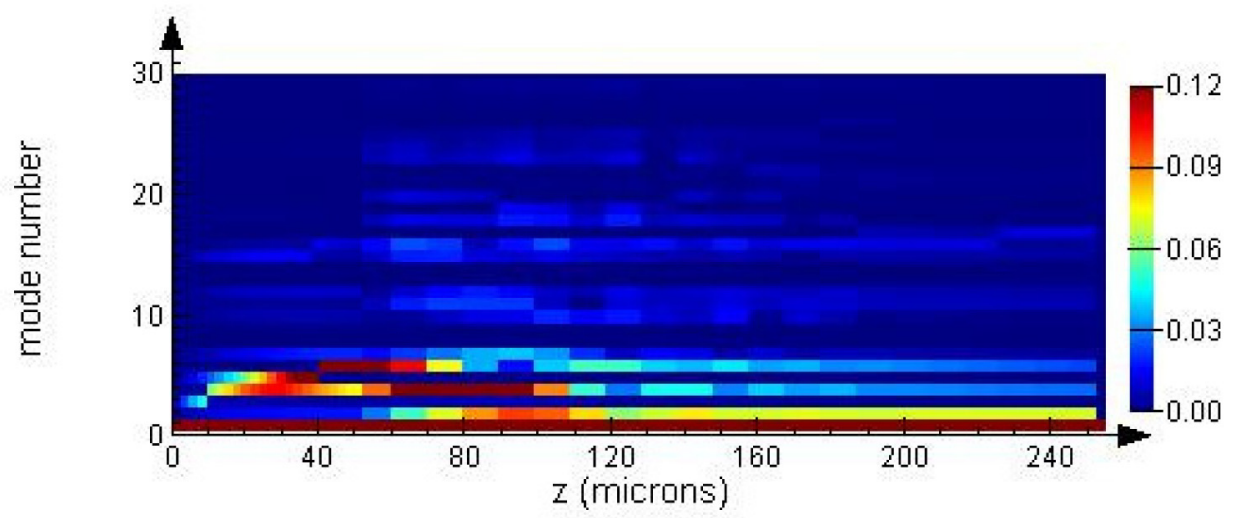

Fig. 9. The magnitude of the forward propagating mode coefficients as a function of position along the length of the edge coupler for the HNA fiber design (Design 1). The color bar is intentionally oversatured to emphasize the magnitudes of the higher order mode coefficients. In this EME simulation, 30 modes were calculated in each cross section.

Similar design procedure was used for Design 2. The structure was too large to be simulated by the 3D-FDTD and we used the 3D-EME calculations. Since an excellent agreement has been demonstrated between the FDTD and EME results (Fig. 8, Design 1, EME and FDTD), it is reasonable to expect similar accuracy when using EME calculations for Design 2. The respective lengths of the three $\mathrm{Si}_{3} \mathrm{~N}_{4}$ layers are $1320 \mu \mathrm{m}$ (bottom), $660 \mu \mathrm{m}$ (middle) and $165 \mu \mathrm{m}$ (top). The calculated electric field evolution along the coupler is shown in Fig. 8(c). The transmission to the fundamental mode as a function of length is shown in Fig. 8(d) (red curve). It is observed that the optimal coupler performance is achieved for the coupling length of $1500 \mu \mathrm{m}$, with coupler transmittance of $90.5 \%$. The overall coupling efficiency including the mode overlap at the facet is $84 \%$. This corresponds to an overall coupling loss of $0.75 \mathrm{~dB}$.

Wavelength dependence of both coupler designs was evaluated by 3D-EME simulations. It was determined that both couplers behave substantially wavelength independent, with coupling efficiency variations of less than $0.2 \mathrm{~dB}$ in the wavelength range $1450 \mathrm{~nm}-1600$ $\mathrm{nm}$, as presented in Fig. 10. These results compare favorably to previously reported spot size converters based on overlays that showed substantially larger coupling efficiency variations [14] or overall coupling loss penalty [17-19].

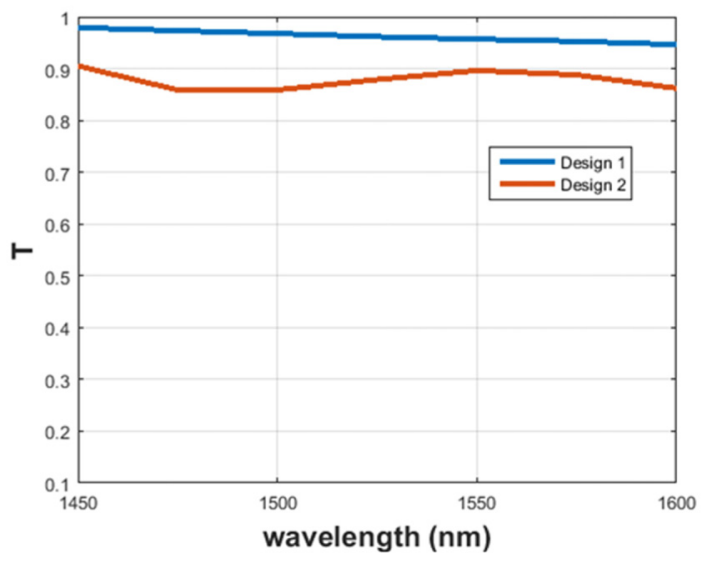

Fig. 10. Wavelength dependence of the two coupler designs. 
Our approach is presented here for the combination of $220 \mathrm{~nm}$ silicon and $3 \mu \mathrm{m}$ thick BOX layers. While $2 \mu \mathrm{m}$ BOX thickness is more common for MPW (multi-project wafer) runs in photonic foundries, importantly our couplers are compatible with the full library of photonic devices available for the $220 \mathrm{~nm}$ silicon platform, with only a change of the BOX thickness required. For our present design $(3 \mu \mathrm{m} \mathrm{BOX})$, the calculated reduction in coupling efficiency for a $2 \mu \mathrm{m}$ BOX is $0.4 \mathrm{~dB}$ (Design 1). The leakage to substrate for $2 \mu \mathrm{m}$ BOX can potentially be reduced by increasing the effective index of the overlay, which can be achieved by adding additional layers and/or increasing their thickness.

\section{Conclusions}

We propose a new type of fiber-chip edge coupler with large mode size with the total coupling efficiency exceeding $90 \%(0.42 \mathrm{~dB})$. The coupler allows direct coupling with conventional cleaved optical fibers with large mode size while circumventing the need for lensed fibers. An important advantage of our coupler is that the mode size is substantially increased while minimizing the substrate leakage loss through the buried oxide. This remarkable property is achieved by increasing the effective index of the upper $\mathrm{SiO}_{2}$ cladding with subwavelength index engineered thin $\mathrm{Si}_{3} \mathrm{~N}_{4}$ layers. Because the mode evolution technique is employed, wavelength dependence is minimized for the coupler. 3D-FDTD results are validated by 3D-EME simulations, yielding an excellent agreement between the two technique. Our results open a promising venue for interfacing silicon nanophotonic circuits directly with standard optical fibers.

\section{Acknowledgments}

This work was supported by the Ministry of Education, Youth and Sports of the Czech Republic through grant project no. CZ.1.07/2.3.00/20.0217 within the Operation Programme Education for Competitiveness financed by the European Structural Funds and from the state budget of the Czech Republic. Support by the National Research Council of Canada and by the Slovak Research and Development Agency (SRDA) under the project APVV-0025-12 is also acknowledged. 